\title{
Non-Contact Vision-Based Cardiopulmonary Monitoring in Different Sleeping Positions
}

\author{
Michael H. Li, Azadeh Yadollahi, and Babak Taati
}

Version Post-Print/Accepted Manuscript

Citation M. Li, A. Yadollahi, and B. Taati. Non-Contact Vision-Based

(published version) Cardiopulmonary Monitoring in Different Sleeping Positions. IEEE

Journal of Biomedical and Health Informatics, 2016 [EPub ahead of print], DOI: 10.1109/JBHI.2016.2567298

Publisher's Statement

C 2016 IEEE. Personal use of this material is permitted. Permission from IEEE must be obtained for all other uses, in any current or future media, including reprinting/republishing this material for advertising or promotional purposes, creating new collective works, for resale or redistribution to servers or lists, or reuse of any copyrighted component of this work in other works.

How to cite TSpace items

Always cite the published version, so the author(s) will receive recognition through services that track citation counts, e.g. Scopus. If you need to cite the page number of the TSpace version (original manuscript or accepted manuscript) because you cannot access the published version, then cite the TSpace version in addition to the published version using the permanent URI (handle) found on the record page. 


\title{
Non-Contact Vision-Based Cardiopulmonary Monitoring in Different Sleeping Positions
}

\author{
Michael H. Li, Student Member, IEEE, Azadeh Yadollahi, Member, IEEE and Babak Taati, Member, \\ IEEE
}

\begin{abstract}
Individuals with obstructive sleep apnea (OSA) can experience partial or complete collapse of the upper airway during sleep. This condition affects between $10-17 \%$ of adult men and 3-9\% of adult women, requiring arousal to resume regular breathing. Frequent arousals disrupt proper sleeping patterns and cause daytime sleepiness. Untreated OSA has been linked to serious medical issues including cardiovascular disease and diabetes. Unfortunately, diagnosis rates are low ( 20\%) and current sleep monitoring options are expensive, time-consuming, and uncomfortable. Towards the development of a convenient, non-contact OSA monitoring system, this paper presents a simple, computer vision-based method to monitor cardiopulmonary signals (respiratory and heart rates) during sleep. System testing was performed with 17 healthy participants in five different simulated sleep positions. To monitor cardiopulmonary rates, distinctive points are automatically detected and tracked in infrared image sequences. Blind source separation is applied to extract candidate signals of interest. The optimal respiratory and heart rates are determined using periodicity measures based on spectral analysis. Estimates were validated by comparison to polysomnography recordings. The system achieved a mean percentage error of $3.4 \%$ and $5.0 \%$ for respiratory rate and heart rate respectively. This study represents an important step in building an accessible, unobtrusive solution for sleep apnea diagnosis.
\end{abstract}

Index Terms - computer vision, sleep apnea, physiological monitoring, cardiopulmonary signals, blind source separation

\section{INTRODUCTION}

Obstructive sleep apnea (OSA) is a sleep breathing disorder characterized by partial or complete obstruction of the upper airway during sleep. These obstructions disrupt respiration, causing arousal to resume breathing, thus preventing the individual from getting adequate deep sleep and severely

This paper was submitted for review on December 15, 2015; revised March 17, 2016 and May 7, 2016; accepted May 10, 2016. This work was supported in part by funding from the Toronto Rehabilitation Institute University Health Network, in part by Natural Sciences and Engineering Research Council of Canada (NSERC Discovery Grant, RGPIN 435653), and in part by BresoTec Inc.

M. H. Li is with the Institute of Biomaterials and Biomedical Engineering, University of Toronto, Toronto, ON M5S3G9 Canada (e-mail: michaelhg.li@mail.utoronto.ca).

A. Yadollahi is with the Institute of Biomaterials and Biomedical Engineering, University of Toronto, Toronto, ON M5S3G9 Canada and the Toronto Rehabilitation Institute - University Health Network, Toronto, ON M5G2A2 Canada (e-mail: azadeh.yadollahi@uhn.ca).

B. Taati is with the Toronto Rehabilitation Institute - University Health Network, Toronto, ON M5G2A2 Canada and with the Department of Computer Science, University of Toronto, Toronto, ON M5S3G4 Canada (email: Babak.Taati@uhn.ca). impacting overall sleep quality. OSA is estimated to affect between $10-17 \%$ of adult men and 3-9\% of adult women [1]. Risk factors for OSA include age, sex, obesity, family history and race [2]. Left untreated, OSA has been linked to an increase in motor vehicle and occupational accidents, hypertension, and cardiovascular disease [3], as well as diabetes [4] and depression [5].

Despite the well-documented consequences of untreated OSA, studies suggest that at least $80 \%$ of individuals with OSA are undiagnosed [6], [7], [8]. The current gold standard for diagnosis is complete laboratory polysomnography (PSG). PSG involves numerous recordings, including electroencephalogram (EEG), electrocardiogram (ECG), and respiratory effort. While PSG provides the most comprehensive information for identifying sleep disordered breathing, it requires an overnight stay at a sleep laboratory. This process is both inconvenient and uncomfortable. PSG availability may be limited depending on geographical location, with wait times for sleep studies in Canada outside Ontario varying between 8-36 months [9]. In the United States, wait times are similarly variable, ranging between a few weeks to 9 months [9]. The cost of PSG may also be a deterrent for sleep testing, as the cost of full-night PSG in the US is over \$600 USD (Medicare Physician Payment Schedule 2015 - National Payment Amount, HCPCS 95810). Therefore, it is clear that conventional sleep assessment methods are inadequate to combat the severe underdiagnosis of OSA. While home sleep assessment may be a more viable alternative, available technologies still rely on contact sensors which can lose connection over the duration of the study or present safety hazards [10].

Within this context, efforts are underway by the authors to develop an inexpensive, non-contact, unobtrusive method of sleep monitoring that imposes minimal restrictions on the user. Methods and results presented here capture respiration and heart rates in various simulated sleeping positions, thus laying important groundwork for OSA monitoring and diagnosis in future work. The non-contact nature is also useful for physiological monitoring of populations with fragile skin, such as neonates or older adults.

Recent advancements in camera technologies and computing power have made it feasible to use computer vision for non-contact monitoring. The proposed system consists only of an infrared (IR) sensitive camera, a source of IR illumination, and a computer to process information. For privacy reasons, camera frames are discarded after the relevant cardiopulmonary signals have been extracted, such that no image is kept for more than 30 seconds. 
This study is an extension of a feasibility study that focused on respiratory rate estimation [11]. Specific contributions of this paper include:

1) Collection and processing of a larger dataset with more participants and sleeping positions;

2) Continuous non-contact monitoring of heart rate;

3) Detailed presentation of algorithmic steps with sufficient information to implement and reproduce presented results; and

4) Extensive experimental results, analysis of failure modes, and identification of future research priorities.

\section{Previous WorK}

Changes in respiratory rate and heart rate are both useful predictors of OSA. Therefore, this review encompasses noncontact methods for respiratory rate estimation, heart rate estimation and apnea detection. Currently, the most popular modalities for these purposes have been computer vision, thermal cameras, and radar.

Computer vision-based monitoring systems detect physiological signals in video by identifying the motions associated with those signals ( "motion-based methods") or by using color or brightness change of the skin due to blood perfusion ("color-based methods"). Motion-based methods use intensity difference or optical flow of consecutive images to extract respiratory and cardiac motion from video. An early implementation averaged intensity difference or optical flow over the entire image to get a global motion signal, which was effective at capturing posture changes and apneas but was not robust to noise [12]. More recent studies have exploited the irregularity of breathing patterns during apneas to identify respiratory events using entropy analysis [13] or by comparing respiration to adaptive templates of normal breathing [14]. To reduce complexity, one approach projected video frames to a one-dimensional profile and analyzed changes of the profile over time to extract respiratory rate [15]. A novel motionbased method used head oscillations caused by blood pumping to the head to quantify heart rate [16]. Color-based methods use brightness changes related to blood perfusion to quantify heart rate and respiratory rate. Initially, skin patches were manually segmented and brightness changes were captured using a consumer video camera [17]. Using face detection and independent component analysis (ICA), a framework was established for automatic segmentation, extraction of the pulse signal, respiratory rate, and heart rate variability [18]. These methods have also been extended for infrared light [19], and have been tested in more realistic environments, such as monitoring of dialysis patients [20]. The central weakness with color-based methods is they require skin regions to be visible and segmented.

Thermal cameras can measure respiration by monitoring changes in airflow temperature [21] or nasal temperature [22], [23], and can measure pulse by monitoring the temperature of superficial facial arteries [24]. Radar has been used to measure chest motion related to respiration and pulse and has the added benefit of being able to permeate certain occlusions, such as clothes or walls. SleepMinder, a radar-based device for sleep monitoring, was able to detect respiratory motion and predict apnea-hypopnea index (AHI) [25].
The feasibility of other non-contact methods such as ultrasonic [26], passive infrared [27], motion capture [28], and magnetic induction [29], have been demonstrated in preliminary studies, but these methods have not gained as much traction as the aforementioned methods. At this time, these methods remain in the proof of concept stage.

In this study, a motion-based computer vision method is presented for cardiopulmonary monitoring. This method presents several advantages over existing methods. Cameras are readily accessible, and video processing can potentially provide a convenient means of identifying sleep position and differentiating multiple persons in the same bed. A motionbased method is preferred over a color-based, since skin detection is difficult or unavailable during sleep. While computer vision methods have been used to extract breathing patterns, to the authors' knowledge, they have not been used to extract heart rate from chest motion without attachment or projection of markers.

\section{MAterials AND MethodS}

\section{A. Participants}

17 participants were recruited for this study ( 10 women, 7 men, age $=29.9 \pm 11.7$ years, $\left.\mathrm{BMI}=22.4 \pm 2.5 \mathrm{~kg} / \mathrm{m}^{2}\right)$. This study was approved by the University Health Network Research Ethics Board and the University of Toronto Research Ethics Board. All participants provided written informed consent before participation. The participants were not specifically screened or selected based on a history of breathing disorders.

\section{B. Measurements}

Data was collected at the Toronto Rehabilitation Institute Sleep Laboratory. Participants lied down on a bed in a dark room to simulate overnight sleeping conditions (Fig. 1). The room was illuminated by an IR light (Raytec RM25-F-50). A camera was placed approximately $1.4 \mathrm{~m}$ above the bed, such that the person's head and torso were captured. The camera model was a Point Grey Firefly MV (0.3 MP, FMVU03MTM) with an IR sensitive image sensor (Micron, MT9V022177ATM). Video was recorded with a resolution of $640 \times 480$ at $30 \mathrm{fps}$.

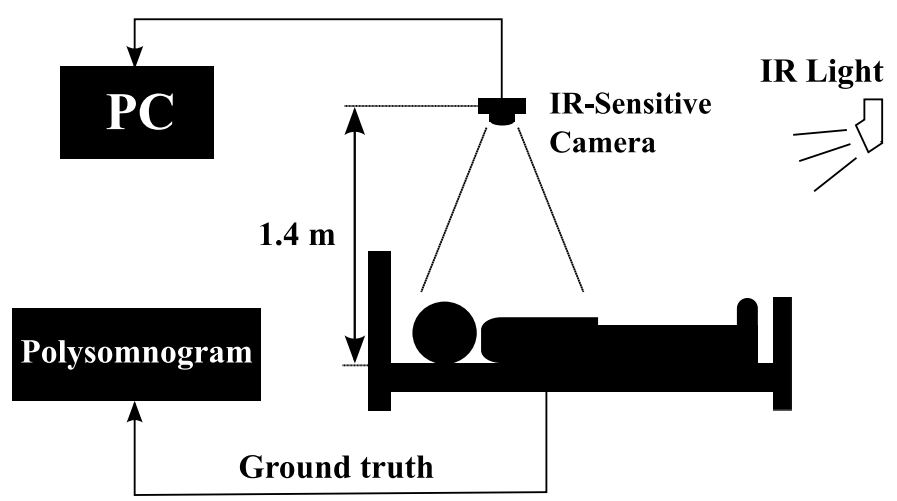

Fig. 1. Schematic of the experimental setup.

Heart rate was measured using a one lead ECG and respiratory motion was measured using respiratory belts 
(inductance plethysmography) placed around the chest and abdomen. These recordings served as the gold standard for validation. Data was recorded with the sleep laboratory's PSG system and Embla Sandman software. The sampling rates for heart rate and respiration were 4 and $20 \mathrm{~Hz}$ respectively.

Participants were asked to lie in five different positions, and to remain still for approximately five minutes (range $4 \mathrm{~m} 47 \mathrm{~s}-$ $6 \mathrm{~m} 23 \mathrm{~s}$, average $5 \mathrm{~m} 29 \mathrm{~s}$ ) in each position. The positions were: lying supine, lying on their left side, lying on their right side, lying prone, and lying supine while the torso was occluded with a sheet (Fig. 2). This study extends a previously conducted feasibility study, following a similar protocol with the only major difference being the addition of the prone position [11].

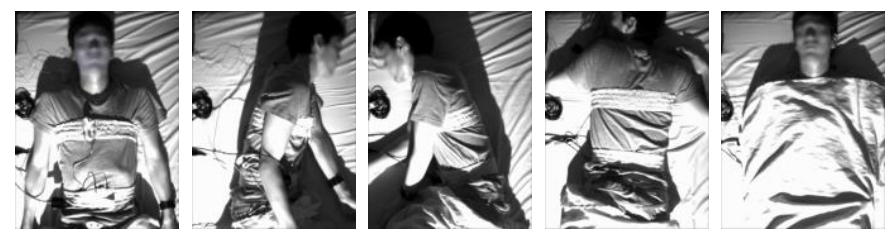

Fig. 2. Different positions used for recording. From left to right: supine, left side, right side, prone, and supine occluded.

\section{Algorithm Overview}

A schematic overview of the algorithm is provided in Fig. 3. Respiratory rate and heart rate are extracted from an IR video using a computer vision-based approach. Feature points are identified in the first frame of the video and tracked over time. The feature point trajectories are processed in order to identify chest motion related to respiration and cardiac function. The trajectories are filtered to remove noise outside the expected signal frequency range. Using blind source separation, the trajectories are reduced to a smaller set of signals representative of the overall motion over a sliding window in time. The signals that most closely resemble expected respiratory and pulse signals are selected. A frequency spectrum analysis informs the estimation of respiratory rate and heart rate. Video analysis was performed offline in Python 2.7 and OpenCV 2.4.6. Detailed discussion of these steps is provided in the following sections.

\section{Motion Tracking and Preprocessing}

To track motion, distinctive points (e.g. corners, textured areas) in the image are identified and tracked over time. It is expected that chest motion will provide the most useful information for respiratory rate and heart rate detection. To avoid image segmentation, points are detected and tracked over the entire image and the characteristics of their trajectories are used to determine their usefulness. First, the image is divided into an $A \times B$ grid of cells. The $P$ most distinctive points are selected in each grid cell using feature point detection. Using a grid ensures that feature points are distributed over the entire image, enabling capture of chest motion regardless of sleeping position.

Feature points are tracked over time using optical flow. The trajectories of all feature points over a window of time are analyzed. For heart rate and respiratory rate estimation, sliding windows of lengths $\tau_{\mathrm{H}}$ and $\tau_{\mathrm{R}}$ respectively are used and a new estimate is computed every second. Fig. 3(a) illustrates a sample frame with the grid superimposed to show the even distribution of points.

In order to isolate relevant motions, the signal qualities of irrelevant trajectories must be considered. Some feature points may have insufficient texture for accurate tracking and will generate erratic trajectories. Another group of feature points will not be on the body and will not have significant motion. To exclude both groups of points, the maximum frame-toframe displacement is determined for each point and the top $M^{\text {th }}$ and bottom $N^{\text {th }}$ percentiles are discarded. Subsequently, motion trajectories are split into their vertical and horizontal trajectories. Horizontal and vertical trajectories with a range of motion less than their respective mean ranges are discarded. Remaining trajectories are pooled for further analysis.

Algorithm and parameter selection are determined empirically considering processing speed, memory usage, and accuracy. The selected grid parameters for feature point detection are $A=12$ and $B=16$, such that each grid cell is a square with a side length of 40 pixels. In each grid cell, $P=8$ feature points are identified for a maximum of 1536 points over the entire image. Feature point detection is performed using Harris corner detection while Lucas-Kanade optical flow is used for motion tracking. The sliding window used for each consecutive estimate of heart rate and respiratory rate is 30 seconds $\left(\tau_{\mathrm{H}}=\tau_{\mathrm{R}}=30 \mathrm{~s}\right)$, yielding an overlap between adjacent windows of 29 seconds or $96.7 \%$. Discarded percentiles $M$ and $N$ are both set to $25 \%$.

\section{E. Respiratory Rate Estimation}

To constrain signal frequencies to the expected range of respiratory rate, a $5^{\text {th }}$ order band pass Butterworth filter is applied with a passband of $[0.1,1] \mathrm{Hz}$ or $6-60$ breaths/minute (Fig. 3(b)). The Butterworth filter is selected due to its flat passband, causing minimal signal distortion. Blind source separation is applied via principal component analysis (PCA), which decomposes the data set into a set of linearly uncorrelated variables. For a set of $k$ feature trajectories, PCA finds $k$ orthogonal trajectories, or principal components. The standard implementation of PCA involves the computation and diagonalization of the sample covariance matrix, operations that become computationally prohibitive for a large number of features. Instead, for computational efficiency, an expectationmaximization implementation (EMPCA) is used [30]. EMPCA is used to iteratively compute the $K_{R}$ principal components that explain the most variance in the data. $K_{R}=5$ is sufficient such that respiratory motion can be consistently recovered in the top $K_{R}$ components while reducing noise that could impact performance.

As blind source separation does not provide interpretation of extracted sources, the periodic nature of respiration is exploited to isolate the principal component that is most similar to a respiratory signal. Spectral estimation is used to investigate signal periodicity. A Hamming window is applied to reduce edge effects and the signal is zero-padded before a Fast Fourier Transform (FFT) is applied. Zero-padding is akin to interpolation in the frequency domain by a factor of $L_{R}$, thus providing a more fine-grained estimate. For this study, $L_{R}=4$. The periodicity is quantified by the peak-to-total ratio - the ratio of the amplitude of the highest peak in the frequency spectrum to the total spectral density. A high periodicity 

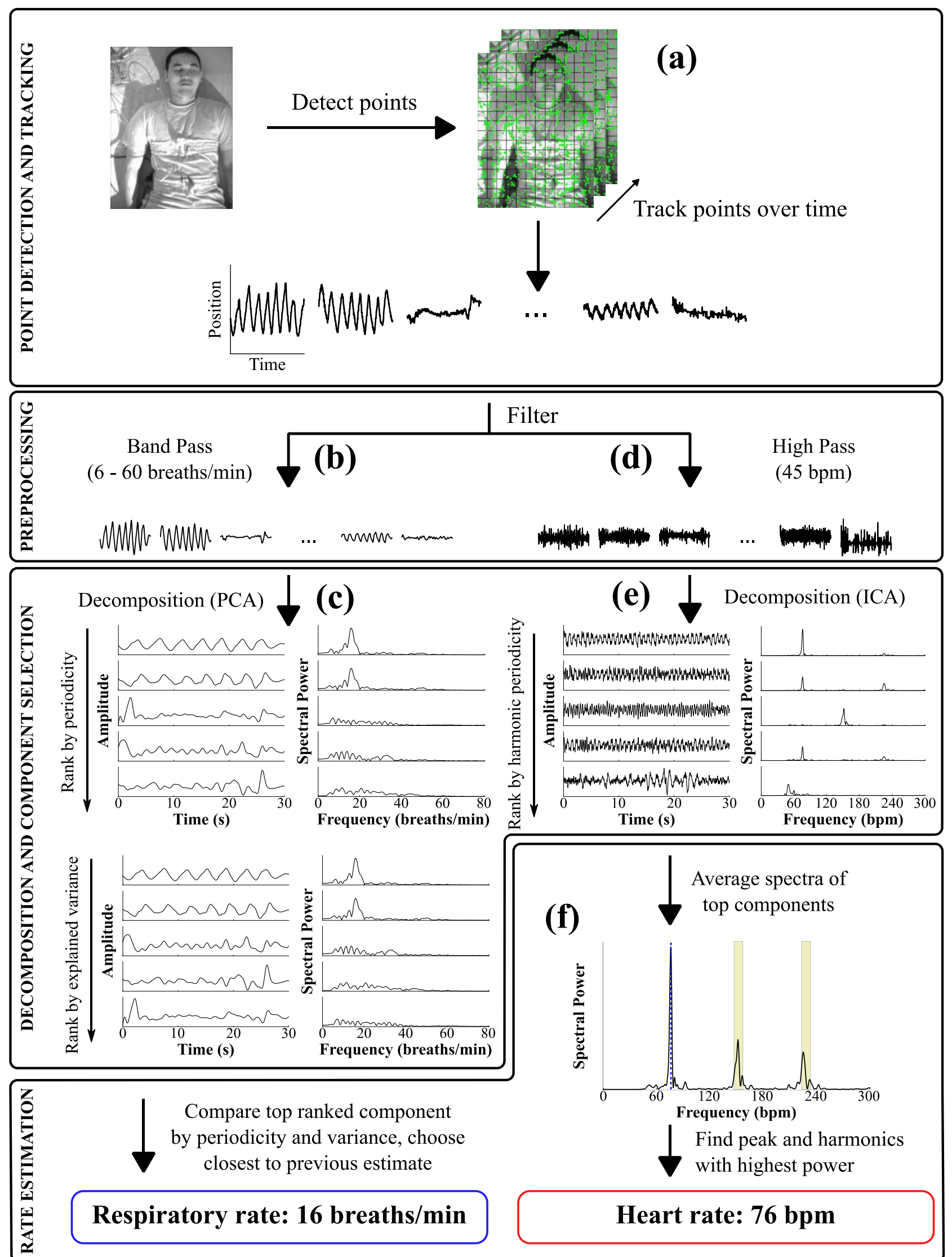

Heart rate: 76 bpm

Fig. 3. Overview of algorithm. (a) Points are detected on video frame and tracked over time. (b), (d) Trajectories are filtered for respiratory rate and heart rate estimation. (c), (e) Trajectories are decomposed into a smaller set of representative components. Components are ranked based on different criteria. For respiratory rate estimation, these rankings and the previous respiratory rate estimate provide sufficient information to estimate the current rate. (f) For heart rate estimation, the frequency spectra of top ranked components are combined to determine the heart rate. 
indicates a dominant frequency while a low periodicity indicates the signal is more likely to correspond to aperiodic noise.

While using the periodicity measure to rank components works well in noiseless conditions, in practice, there are perturbations that complicate respiratory rate estimation. The proposed method incorporates periodicity, explained variance, and temporal continuity for accurate component selection. Fig. 3(c) shows the top five principal components ranked by periodicity as well as explained variance. Small voluntary motions (e.g. adjusting position, turning head) or low amplitude periodic contaminants (e.g. vibration, electronic noise) can affect signal decomposition, increasing the difficulty of component selection. To impose smoothness of consecutive estimates over time, closeness to previous estimates was used to select the best component. The dominant frequency (i.e. the peak of the frequency spectrum) is found for the most periodic component and the component with the highest explained variance. The frequency closer to the previous estimate is selected as the respiratory rate estimate. For the initial estimate, the component with the highest variance is selected. This is sensible since voluntary motions are easier to manually exclude than periodic contaminants, which may not be visible to the naked eye. There are two important assumptions made in this approach: that the first $\tau_{\mathrm{R}}$ seconds of the recording do not contain motion artifacts (such that the first estimate is accurate), and that changes in respiratory rate are slow relative to the rate at which the estimate is updated (once per second).

\section{F. Heart Rate Estimation}

While heart rate estimation follows a similar framework to respiratory rate estimation, estimating heart rate is inherently more difficult than estimating respiratory rate since pulse related motions have smaller amplitude. Previous studies have noted that respiratory rate harmonics can interfere with the heart rate fundamental frequency [31], [32]. Therefore, heart rate estimation employs more complex approaches to yield accurate estimation.

Signals are passed through a $5^{\text {th }}$ order, high pass Butterworth filter with a cutoff frequency of $0.75 \mathrm{~Hz}$ or 45 bpm (beats per minute) (Fig. 3(d)). Blind source separation is performed using ICA. The FastICA implementation is used for its rapid convergence speed and ease of use. ICA components are iteratively selected such that each consecutive source's independence is maximized. This process is repeated to extract $K_{H}$ independent components. Choosing $K_{H}=10$ is suitable to capture the heart rate fundamental frequency and harmonics while limiting noise that can impact estimation accuracy.

Once the independent components are extracted, they are ranked by their relative importance for heart rate estimation. Unlike PCA, ICA does not provide a readily available importance measure like explained variance in the original dataset. Using the peak-to-total ratio as a periodicity measure is insufficient for accurate estimation as respiratory harmonics can be in the same frequency range. Instead, a harmonic periodicity measure is defined as the sum of the amplitudes of the fundamental frequency and its first two harmonics divided by the total spectral density. Before computing the frequency spectrum, a Hamming window is applied and the spectrum is interpolated by a factor $L_{H}$ using zero-padding, where $L_{H}$ is the same as for respiratory rate $\left(L_{H}=4\right)$. An FFT is used for spectral estimation, after which the spectrum is squared to increase the amplitude of peaks relative to noise. Peak detection is used to find candidates for the fundamental frequency and harmonics. A minimum peak height $H$ and maximum number of peaks $\beta$ are defined to ensure the quality and limit the number of peaks identified. To match peaks with their harmonics, search windows are used where the harmonics are expected. The width of the search window is defined by the frequency tolerance $\omega$. For example, a peak at $60 \mathrm{bpm}$ will have search windows at $120 \pm \omega \mathrm{bpm}$ and $180 \pm$ $\omega \mathrm{bpm}$ for its first and second harmonics respectively. The candidates for the fundamental frequency are restricted to be less than $180 \mathrm{bpm}$ to reject high frequency noise and because heart rate is not expected to exceed $180 \mathrm{bpm}$ during sleep. The harmonic periodicity of the component is given by the highest harmonic periodicity of the detected peaks in the component's spectrum. Setting the minimum peak height $H$ to the 90th percentile of the spectrum and the maximum number of peaks $\beta=12$ allows the heart rate fundamental and its harmonics to be identified without selecting spurious peaks. The frequency tolerance is set to 5 ( $\omega=5 \mathrm{bpm}$ ), making the search window small enough to reject unrelated peaks but still wide enough to detect true harmonics.

Fig. 3(e) shows the top independent components ranked by harmonic periodicity. Components with a harmonic periodicity less than the mean are discarded. The spectra of the remaining components are normalized and averaged to form a combined spectrum. The harmonic periodicity is computed for the peaks of the combined spectrum and the peak with the highest harmonic periodicity is the heart rate estimate. Fig. 3(f) shows the averaged spectrum, where the dotted line is the fundamental frequency and the shaded regions are the search windows for harmonics. To prevent sudden fluctuations in subsequent estimates, the combined spectrum is averaged with the combined spectrum from the previous window (temporal smoothing) before performing peak detection.

\section{G. System Evaluation}

The respiratory information from the PSG is a respiratory trace, which is a signal showing expansion and contraction of the chest and abdomen over time. The ground truth respiratory rate is extracted from the trace by using the same filter, windowing and Fourier transform as for the vision-based system. The respiratory rate is taken as the frequency corresponding to the global peak in the frequency spectrum. The ground truth heart rate is directly provided from the sleep lab's PSG system.

Metrics for respiratory rate estimation are root-mean-square (RMS) error, mean percentage error (MPE) and percentage of estimates within 1.0 and 0.5 breaths/minute of the true respiratory rate. Metrics for heart rate estimation are RMS error, MPE and percentage of estimates within 5 and $2.5 \mathrm{bpm}$ of the true heart rate.

\section{RESULTS}

There were a total of 75 trials recorded. Data from six of the participants was gathered as part of a pilot study [11]. 
However, the pilot study did not include recordings in the prone position. Therefore, prone position recordings are only available for the 11 newly recorded participants. The remaining trials that were not recorded were due to technical difficulties. For heart rate, one of the subjects was excluded (for all five positions) due to an irregular heartbeat, which is outside the scope of our analysis. Full results for each participant with details regarding excluded trials are included in the Supplementary Material.

Results are presented in Table I. The average MPE for respiratory rate detection over all positions was $3.4 \%$ and estimation was accurate within 1.0 breaths/min for $94.4 \%$ of the times. The average MPE for heart rate estimation over all positions was $5.0 \%$ and estimation was accurate within $5 \mathrm{bpm}$ for $87.0 \%$ of the times. Averages and standard deviations (SD) are weighted such that each position is treated equally.

TABLE I

RESULTS FOR RESPIRATORY RATE AND HEART RATE ESTIMATION

\begin{tabular}{|c|c|c|c|c|c|c|}
\hline $\begin{array}{r}\text { Respiratory } \\
\text { Rate }(n=75)\end{array}$ & $\begin{array}{l}\text { Supine } \\
(n=17)\end{array}$ & $\begin{array}{c}\text { Left } \\
(n=16)\end{array}$ & $\begin{array}{l}\text { Right } \\
(n=15)\end{array}$ & $\begin{array}{l}\text { Prone } \\
(n=11)\end{array}$ & $\begin{array}{l}\text { Occluded } \\
(n=16)\end{array}$ & $\begin{array}{l}\text { Average } \\
\text { (SD) }\end{array}$ \\
\hline $\begin{array}{r}\text { RMS Error } \\
\text { (breaths/min) }\end{array}$ & 0.86 & 1.14 & 1.36 & 0.92 & 1.33 & $\begin{array}{c}\mathbf{1 . 1 2} \\
(1.38)\end{array}$ \\
\hline $\begin{array}{r}\% \pm<1.0 \\
\text { breaths/min }\end{array}$ & $95.6 \%$ & $94.1 \%$ & $92.1 \%$ & $96.0 \%$ & $94.5 \%$ & $\begin{array}{l}\mathbf{9 4 . 4 \%} \\
(8.6 \%)\end{array}$ \\
\hline $\begin{array}{r}\% \pm<0.5 \\
\text { breaths/min }\end{array}$ & $93.2 \%$ & $92.6 \%$ & $87.2 \%$ & $93.4 \%$ & $91.3 \%$ & $\begin{array}{l}\mathbf{9 1 . 5 \%} \\
(10.0 \%)\end{array}$ \\
\hline MPE & $2.8 \%$ & $3.4 \%$ & $4.3 \%$ & $2.7 \%$ & $3.6 \%$ & $\begin{array}{l}\mathbf{3 . 4 \%} \\
(3.9 \%)\end{array}$ \\
\hline $\begin{array}{r}\text { Heart Rate } \\
(n=70)\end{array}$ & $\begin{array}{l}\text { Supine } \\
(n=16)\end{array}$ & $\begin{array}{c}\text { Left } \\
(n=15)\end{array}$ & $\begin{array}{c}\text { Right } \\
(n=14)\end{array}$ & $\begin{array}{l}\text { Prone } \\
(n=10)\end{array}$ & $\begin{array}{l}\text { Occluded } \\
(n=15)\end{array}$ & $\begin{array}{l}\text { Average } \\
\text { (SD) }\end{array}$ \\
\hline $\begin{array}{r}\text { RMS Error } \\
(\mathrm{bpm})\end{array}$ & 3.52 & 4.47 & 8.48 & 9.14 & 3.75 & $\begin{array}{c}\mathbf{5 . 8 7} \\
(6.42)\end{array}$ \\
\hline $\begin{array}{r}\% \pm<5 \\
\text { bpm }\end{array}$ & $94.5 \%$ & $91.8 \%$ & $81.0 \%$ & $75.8 \%$ & $92.1 \%$ & $\begin{array}{l}\mathbf{8 7 . 0 \%} \\
(19.9 \%)\end{array}$ \\
\hline $\begin{array}{r}\% \pm<2.5 \\
\text { bpm }\end{array}$ & $88.8 \%$ & $87.9 \%$ & $74.0 \%$ & $69.0 \%$ & $81.6 \%$ & $\begin{array}{l}\mathbf{8 0 . 1 \%} \\
(21.7 \%)\end{array}$ \\
\hline MPE & $3.1 \%$ & $3.5 \%$ & $7.2 \%$ & $7.9 \%$ & $3.5 \%$ & $\begin{array}{l}\mathbf{5 . 0 \%} \\
(6.1 \%)\end{array}$ \\
\hline
\end{tabular}

Fig. 4 shows examples of (a) respiratory rate (participant $\# 10$ - prone) and (b) heart rate (participant \#12 - supine) estimation compared to ground truth PSG. The system reliably tracks the changes in cardiopulmonary activity over the course of the trial. Fig. 5 compares the performance of the system in each position across different metrics. When comparing the mean to median in each metric, it is evident that there are outlying trials that drag down the average system performance.

To check if performance differed significantly between positions, a Kruskal-Wallis statistical test was performed with follow-up test to compare mean ranks. Asterisks $(*)$ indicate if there was a statistically significant difference. No significant differences were found between positions for respiratory rate estimation for all of the metrics used. In contrast, a significant difference ( $p<0.0263$ ) was found between the supine and prone positions for heart rate estimation for all metrics except $\%< \pm 2.5 \mathrm{bpm}$. (a)
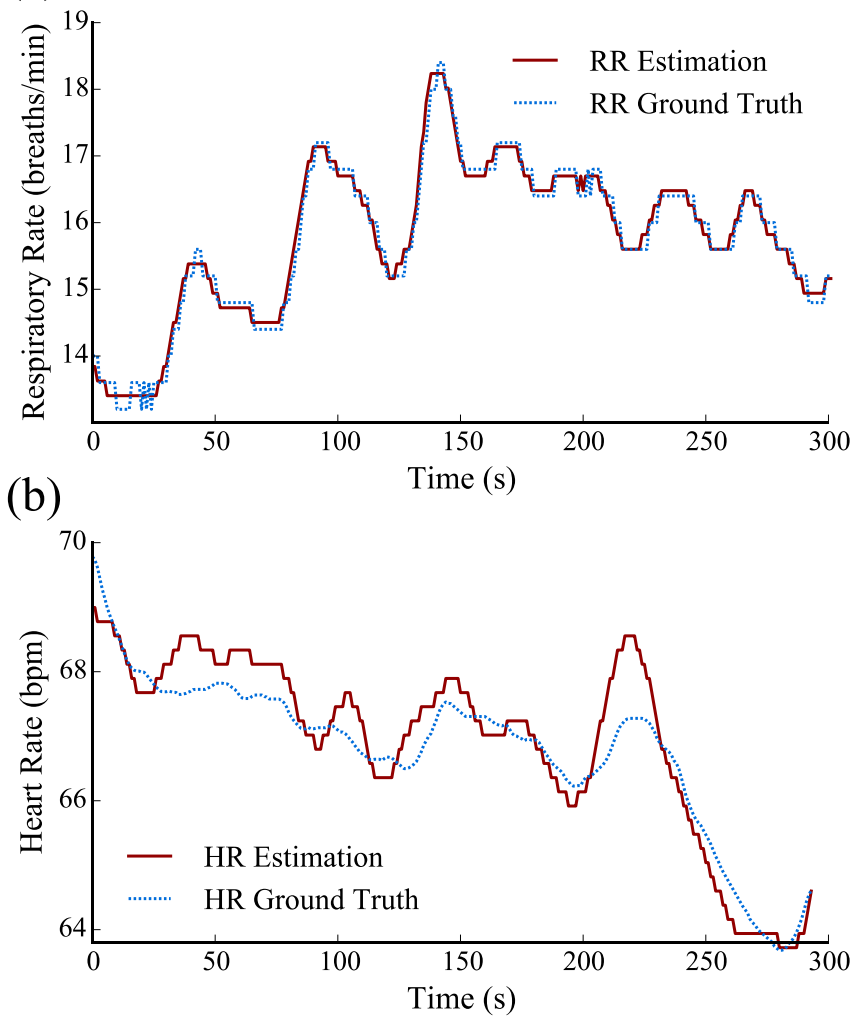

Fig. 4. Examples of system performance for (a) respiratory rate and (b) heart rate.

Table II presents a comparison of performance between the proposed method and two existing methods. For respiratory rate, the existing method extracts respiratory motion using averaged optical flow velocity, as described in [12]. However, the implementation here uses the more recent, publically available, Farnebäck dense optical flow algorithm [33]. For heart rate, the proposed method is compared to Balakrishnan et al. [16]. Since Balakrishnan et al. is used for head motion detection, it is tested both for when the region of interest (ROI) is the full frame of the video and a manually annotated head region.

TABLE II

PERFORMANCE COMPARISON WITH EXISTING METHODS

\begin{tabular}{r|cccc}
\hline \hline Respiratory Rate & $\begin{array}{c}\text { RMS Error } \\
\text { (breaths/min) }\end{array}$ & $\begin{array}{c}\% \pm<1.0 \\
\text { breaths/min }\end{array}$ & $\begin{array}{c}\% \pm<0.5 \\
\text { breaths/min }\end{array}$ & MPE \\
\hline Proposed method & $\mathbf{1 . 1 2}$ & $\mathbf{9 4 . 4 \%}$ & $\mathbf{9 1 . 5 \%}$ & $\mathbf{3 . 4 \%}$ \\
Farnebäck & 4.61 & $74.1 \%$ & $65.8 \%$ & $19.1 \%$ \\
\hline \hline Heart Rate & $\begin{array}{c}\text { RMS Error } \\
\text { (bpm) }\end{array}$ & $\begin{array}{c}\% \pm<5 \\
\text { bpm }\end{array}$ & $\begin{array}{c}\% \pm<2.5 \\
\text { bpm }\end{array}$ & MPE \\
\hline $\begin{array}{r}\text { Proposed method } \\
\text { Balakrishnan } \text { et al. } \\
\text { (full frame) }\end{array}$ & $\mathbf{5 . 8 7}$ & $\mathbf{8 7 . 0 \%}$ & $\mathbf{8 0 . 1 \%}$ & $\mathbf{5 . 0 \%}$ \\
$\begin{array}{r}\text { Balakrishnan } \text { et al. } \\
\text { (head ROI) }\end{array}$ & 39.87 & $58.8 \%$ & $52.7 \%$ & $51.3 \%$ \\
\hline \hline
\end{tabular}

The proposed method significantly outperforms the existing methods in both respiratory rate and heart rate estimation. 
(a)

(b)
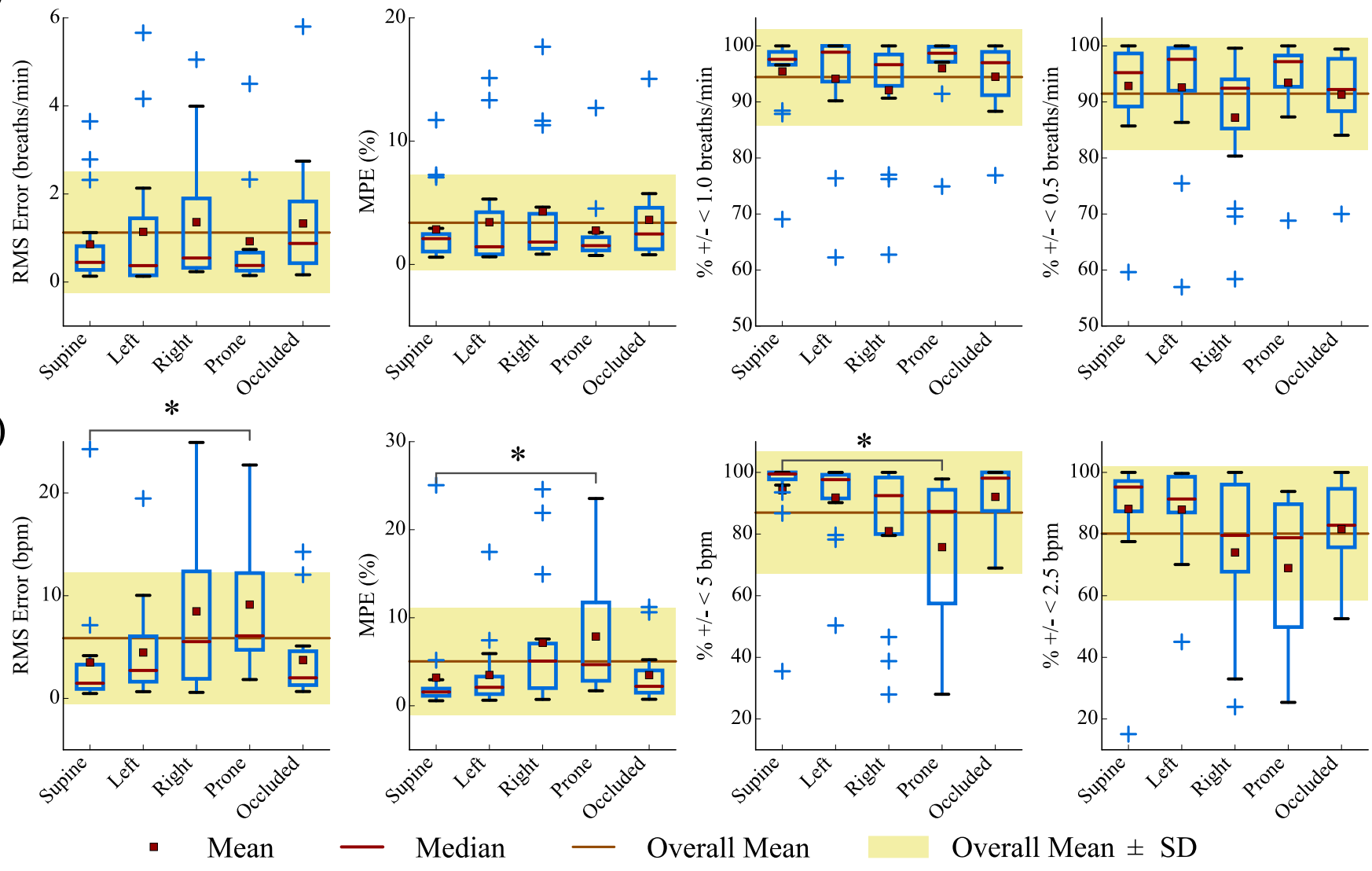

Fig. 5. Comparison of (a) respiratory rate and (b) heart rate estimation results across different positions for different metrics. The box shows the 1st and 3rd quartiles while the whiskers extend to a maximum of 1.5 times the interquartile range.

\section{DISCUSSION}

Statistical testing results indicate that respiratory rate estimation accuracy was consistent in all tested sleeping positions, while heart rate estimation in the prone position was significantly worse than supine position. A study of abdominal surface motion induced by heartbeat supports this finding, as dorsal motion had a lesser magnitude than ventral motion [34]. The prone position is also not a commonly preferred sleep position, with one population study putting prevalence of prone sleepers at only $11 \%$ [35]. The lack of significant difference in heart rate estimation for the metric $\%< \pm 2.5$ bpm may indicate that $2.5 \mathrm{bpm}$ is too stringent of a performance criterion, such that performance for all positions deteriorates. Past evaluations of heart rate monitors have used an agreement within $5 \mathrm{bpm}$ of ECG as a standard for validity [36].

While the video recordings were generally free of complications seriously affecting system performance, a small subset of trials were identified where unexpected issues influenced results (see Supplementary Material). Although participants were asked to lie still during recording to simulate sleeping conditions, there were unwanted motions that occasionally occurred. Consequently, estimation accuracy was impacted for up to $\tau_{\mathrm{R}} / \tau_{\mathrm{H}}$ seconds (30 seconds) from the occurrence of motion. In Fig. 6(a) respiratory rate and heart rate estimation are shown in a sample segment (participant $\# 10$ - left). The shaded area shows the onset and lingering impact of an unwanted motion that occurred around 100 seconds from the start of the trial. Respiratory rate and heart rate estimation are simultaneously impacted. These unwanted motions included yawning or readjustment of arms or torso. The true physiological signal could not be identified in the presence of these motions. While respiratory rate estimation was affected due to the motion being in a similar frequency range, heart rate estimation was also affected despite applying a filter to remove lower frequencies. It is suspected that the amplitude of these unwanted motions was high enough to disturb tracking of the relatively small heartbeat motion. During trials, participants were given no explicit instruction on how to position their arms and legs. In one trial (participant \#5 - right), the participant's arm and shoulder blocked the chest area, leading to poor heart rate estimation. For another participant (participant \#8), heart rate estimation was poor in all positions. While it is speculated that the participant's anatomy was a factor for poor performance, further analysis is required before any conclusions can be drawn.

The camera recording software created some difficulties due to automatic gain, brightness and exposure adjustment. This occasionally introduced high frequency strobing in the video, which affected heart rate estimation. Respiratory rate estimation was unaffected as the strobing was not within the passband of the filter. The impact of this strobing on heart rate estimation appears in Fig. 6(b), where an artificial high frequency was present in the shaded area. This camera software setup problem was corrected after the first five participants were recorded. 
(a)

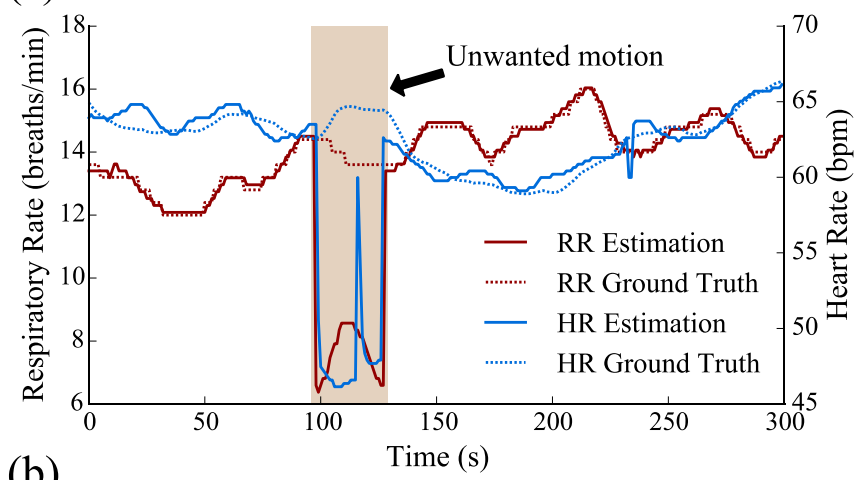

(b)

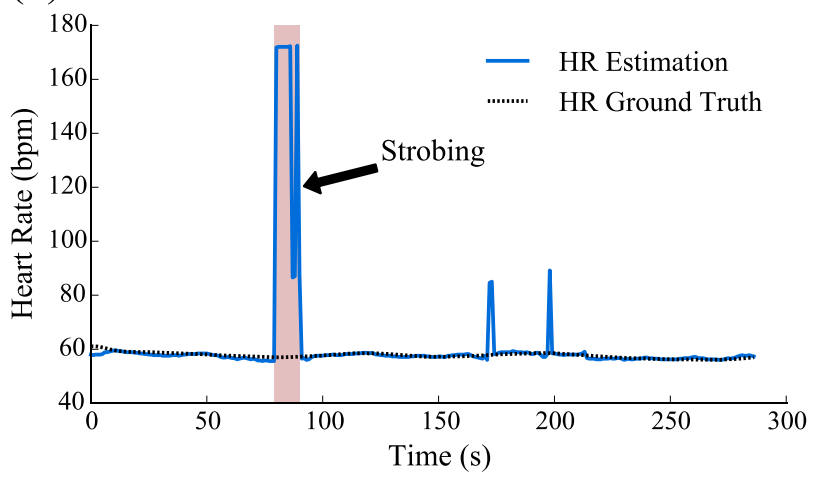

Fig. 6. The impact of (a) unwanted motions and (b) strobing on heart rate (HR) and respiratory rate (RR) estimation.

In order to better illustrate the performance of the system under ideal conditions, a set of results is also presented where the outlying trials mentioned above have been removed (Table III). While it is acknowledged that these results may present a biased representation of system performance, it is expected that motions will be minimal during sleep and camera parameters will be set properly. As such, presenting results both with (Table I) and without (Table III) artifacts provides a more complete picture of anticipated performance.

The discrepancy of performance between the proposed method and the evaluated existing methods can be attributed to several factors. Both existing methods do not incorporate any temporal smoothing and can be prone to sudden changes in estimation. They also have poor resistance to noise and both respiratory and heart rate harmonics. While Balakrishnan et al. includes the 1st harmonic in their periodicity measure, they are not able to distinguish higher harmonics, nor are they able to determine if a peak in the frequency spectrum is the fundamental frequency or a harmonic. Balakrishnan et al. applied to the full frame had a higher $\% \pm<5 \mathrm{bpm}$ than for the head ROI. This may have been because the head motions were damped by the mattress, as opposed to their original experiments where the individual was seated upright in front of a webcam. However, the RMS error for the head ROI was lower than for the full frame ROI. It is believed that harmonics were more prevalent in the full frame ROI due to the inclusion of the chest area, and therefore incorrect estimates were often a multiple of the true rate. This yielded a larger error than an incorrect estimate caused by random noise.
TABLE III

RESULTS FOR RESPIRATORY RATE AND HEART RATE ESTIMATION WITH OUTLIERS REMOVED

\begin{tabular}{|c|c|c|c|c|c|c|}
\hline $\begin{array}{r}\text { Respiratory } \\
\text { Rate }(n=59)\end{array}$ & $\begin{array}{l}\text { Supine } \\
(n=14)\end{array}$ & $\begin{array}{l}\text { Left } \\
(n=13)\end{array}$ & $\begin{array}{l}\text { Right } \\
(n=11)\end{array}$ & $\begin{array}{l}\text { Prone } \\
(n=9)\end{array}$ & $\begin{array}{l}\text { Occluded } \\
(n=12)\end{array}$ & $\begin{array}{c}\text { Average } \\
\text { (SD) }\end{array}$ \\
\hline $\begin{array}{r}\text { RMS Error } \\
\text { (breaths } / \mathrm{min} \text { ) }\end{array}$ & 0.42 & 0.50 & 0.59 & 0.37 & 0.77 & $\begin{array}{c}\mathbf{0 . 5 3} \\
(0.48)\end{array}$ \\
\hline $\begin{array}{r}\% \pm<1.0 \\
\text { breaths/min }\end{array}$ & $98.4 \%$ & $98.3 \%$ & $97.7 \%$ & $98.9 \%$ & $96.9 \%$ & $\begin{array}{l}\mathbf{9 8 . 0 \%} \\
(2.3 \%)\end{array}$ \\
\hline $\begin{array}{r}\% \pm<0.5 \\
\text { breaths/min }\end{array}$ & $96.2 \%$ & $96.8 \%$ & $93.6 \%$ & $96.9 \%$ & $93.7 \%$ & $\begin{array}{l}\mathbf{9 5 . 4 \%} \\
(4.1 \%)\end{array}$ \\
\hline MPE & $1.6 \%$ & $1.6 \%$ & $1.7 \%$ & $1.4 \%$ & $2.3 \%$ & $\begin{array}{l}\mathbf{1 . 7 \%} \\
(1.1 \%)\end{array}$ \\
\hline $\begin{array}{r}\text { Heart Rate } \\
(n=54)\end{array}$ & $\begin{array}{l}\text { Supine } \\
(n=14)\end{array}$ & $\begin{array}{c}\text { Left } \\
(n=11)\end{array}$ & $\begin{array}{l}\text { Right } \\
(n=8)\end{array}$ & $\begin{array}{l}\text { Prone } \\
(n=9)\end{array}$ & $\begin{array}{c}\text { Occluded } \\
(n=12)\end{array}$ & $\begin{array}{c}\text { Average } \\
\text { (SD) }\end{array}$ \\
\hline $\begin{array}{r}\text { RMS Error } \\
(\mathrm{bpm})\end{array}$ & 1.78 & 2.31 & 2.96 & 6.41 & 2.30 & $\begin{array}{c}\mathbf{3 . 1 5} \\
(2.71)\end{array}$ \\
\hline $\begin{array}{r}\% \pm<5 \\
\mathrm{bpm}\end{array}$ & $99.0 \%$ & $96.7 \%$ & $95.5 \%$ & $85.1 \%$ & $95.5 \%$ & $\begin{array}{l}\mathbf{9 4 . 4 \%} \\
(10.5 \%)\end{array}$ \\
\hline $\begin{array}{r}\% \pm<2.5 \\
\mathrm{bpm}\end{array}$ & $94.4 \%$ & $92.5 \%$ & $88.0 \%$ & $77.8 \%$ & $86.0 \%$ & $\begin{array}{c}\mathbf{8 7 . 7 \%} \\
(13.6 \%)\end{array}$ \\
\hline MPE & $1.5 \%$ & $1.9 \%$ & $2.5 \%$ & $4.8 \%$ & $2.3 \%$ & $\begin{array}{l}\mathbf{2 . 6 \%} \\
(2.3 \%)\end{array}$ \\
\hline
\end{tabular}

In experiments with half the video resolution and frame rate (320x240, $15 \mathrm{fps}$ ), minor decreases in performance were noted (respiratory rate - RMS error: +0.1 breaths/min, MPE: $+0.5 \%$; heart rate - RMS error: +1.45 bpm, MPE: $+2.7 \%$ ). However, performance was still superior to existing methods. The run time for the offline analysis was $\sim 38 \mathrm{~ms}$ per frame (with an Intel ${ }^{\circledR}$ Xeon ${ }^{\circledR}$ CPU E3-1240 V2, $3.40 \mathrm{GHz}$ ), which is equivalent to real-time performance at $26.6 \mathrm{fps}$.

A sensitivity analysis (see Supplementary Material) shows that the algorithm achieves comparable performance with a wide range of parameter values, and that further optimization can yield faster and more accurate performance.

\section{A. Limitations}

While this study illustrated the ability to detect physiological signals in simulated sleep situations, system performance may change on individuals who are asleep. In OSA, there will still be respiratory effort despite obstruction. It is unclear how the periodicity measures will be affected and whether the disturbance will be sufficient to classify apneas. Motion tracking with optical flow may suffer if regions lack texture. In practice, this study shows that this is not an issue, even when the subject is covered by an unpatterned white sheet. Provided the camera sensor is not saturated, a white sheet offers enough texture in its creases to support motion extraction. However, the material used to cover the person must be compliant to transfer the chest motion. The current algorithm assumes that there is only one person sleeping in the bed. While this is inconvenient for individuals with a partner, it is still more convenient to sleep alone at home than in a sleep laboratory. Self-occlusion, unwanted motions during sleep (e.g. rolling), and other sleep disorders, such as restless leg syndrome, can also impact the accuracy of estimation, and further investigation is required to circumvent these issues. 


\section{B. Future Directions}

In future studies, video recording will take place on sleeping participants. Methods for automatic sleep position identification and multiple person detection will be explored. To handle unwanted motions, adaptive filtering or constrained ICA can be used to extract physiological signals. Time points where motion occurs can also be demarcated using a simple threshold on total optical flow. Detection of naturally occurring motions during sleep could also potentially be useful as a predictor of sleep state. Additional cameras can be added to the system in order to handle self-occlusion. Once the system has been validated in overnight studies, participants with sleep apnea will be recruited and machine learning techniques will be applied to recognize sleep apnea episodes and to estimate AHI.

\section{REFERENCES}

[1] P. E. Peppard, T. Young, J. H. Barnet, M. Palta, E. W. Hagen, and K. M. Hla, "Increased Prevalence of Sleep-Disordered Breathing in Adults," Am J Epidemiol, vol. 177, no. 9, pp. 1006-1014, May 2013.

[2] N. M. Punjabi, "The Epidemiology of Adult Obstructive Sleep Apnea," Proc Am Thorac Soc, vol. 5, no. 2, pp. 136-143, Feb. 2008.

[3] A. Malhotra and D. P. White, "Obstructive sleep apnoea," The Lancet, vol. 360 , no. 9328 , pp. 237-245, Jul. 2002.

[4] K. J. Reichmuth, D. Austin, J. B. Skatrud, and T. Young, "Association of Sleep Apnea and Type II Diabetes," Am J Respir Crit Care Med, vol. 172, no. 12, pp. 1590-1595, Dec. 2005.

[5] M. M. Ohayon, "The effects of breathing-related sleep disorders on mood disturbances in the general population," J Clin Psychiatry, vol. 64, no. 10, pp. 1195-1200; quiz, 1274-1276, Oct. 2003.

[6] C. Fuhrman, B. Fleury, X.-L. Nguyên, and M.-C. Delmas, "Symptoms of sleep apnea syndrome: High prevalence and underdiagnosis in the French population," Sleep Med, vol. 13, no. 7, pp. 852-858, Aug. 2012.

[7] V. Kapur, K. P. Strohl, S. Redline, C. Iber, G. O'Connor, and J. Nieto, "Underdiagnosis of Sleep Apnea Syndrome in U.S. Communities," Sleep Breath, vol. 6, no. 2, pp. 49-54, Apr. 2002.

[8] T. Young, L. Evans, L. Finn, and M. Palta, "Estimation of the clinically diagnosed proportion of sleep apnea syndrome in middle-aged men and women," Sleep, vol. 20, no. 9, pp. 705-706, Sep. 1997.

[9] W. W. Flemons, N. J. Douglas, S. T. Kuna, D. O. Rodenstein, and J. Wheatley, "Access to Diagnosis and Treatment of Patients with Suspected Sleep Apnea," Am J Respir Crit Care Med, vol. 169, no. 6, pp. 668-672, Mar. 2004.

[10] N. A. Collop, W. M. Anderson, B. Boehlecke, D. Claman, R. Goldberg, D. J. Gottlieb, D. Hudgel, M. Sateia, R. Schwab, and Portable Monitoring Task Force of the American Academy of Sleep Medicine, "Clinical guidelines for the use of unattended portable monitors in the diagnosis of obstructive sleep apnea in adult patients. Portable Monitoring Task Force of the American Academy of Sleep Medicine," $J$ Clin Sleep Med, vol. 3, no. 7, pp. 737-747, Dec. 2007.

[11] M. H. Li, A. Yadollahi, and B. Taati, "A non-contact vision-based system for respiratory rate estimation," in 2014 36th Annual International Conference of the IEEE Engineering in Medicine and Biology Society (EMBC), 2014, pp. 2119-2122.

[12] K. Nakajima, Y. Matsumoto, and T. Tamura, "Development of real-time image sequence analysis for evaluating posture change and respiratory rate of a subject in bed," Physiol Meas, vol. 22, no. 3, pp. N21-N28, Aug. 2001.

[13] E. Gederi and G. D. Clifford, "Fusion of image and signal processing for the detection of obstructive sleep apnea," in 2012 IEEE-EMBS International Conference on Biomedical and Health Informatics (BHI), 2012, pp. 890-893.

[14] C.-W. Wang, A. Hunter, N. Gravill, and S. Matusiewicz, "Unconstrained Video Monitoring of Breathing Behavior and Application to Diagnosis of Sleep Apnea," IEEE Trans Biomed Eng, vol. 61, no. 2, pp. 396-404, Feb. 2014.

[15] M. Bartula, T. Tigges, and J. Muehlsteff, "Camera-based system for contactless monitoring of respiration," in 2013 35th Annual International
Conference of the IEEE Engineering in Medicine and Biology Society (EMBC), 2013, pp. 2672-2675.

[16] G. Balakrishnan, F. Durand, and J. Guttag, "Detecting Pulse from Head Motions in Video," in 2013 IEEE Conference on Computer Vision and Pattern Recognition (CVPR), 2013, pp. 3430-3437.

[17] C. Takano and Y. Ohta, "Heart rate measurement based on a time-lapse image," Med Eng Phys, vol. 29, no. 8, pp. 853-857, Oct. 2007.

[18] M.-Z. Poh, D. J. McDuff, and R. W. Picard, "Advancements in Noncontact, Multiparameter Physiological Measurements Using a Webcam," IEEE Trans Biomed Eng, vol. 58, no. 1, pp. 7-11, Jan. 2011.

[19] F. Zhao, M. Li, Y. Qian, and J. Z. Tsien, "Remote Measurements of Heart and Respiration Rates for Telemedicine," PLOS ONE, vol. 8, no. 10, pp. 1-14, Oct. 2013. doi: 10.1371/journal.pone.0071384

[20] L. Tarassenko, M. Villarroel, A. Guazzi, J. Jorge, D. A. Clifton, and C. Pugh, "Non-contact video-based vital sign monitoring using ambient light and auto-regressive models," Physiol Meas, vol. 35, no. 5, p. 807831, May 2014.

[21] R. Murthy and I. Pavlidis, "Noncontact measurement of breathing function," IEEE Engineering in Medicine and Biology Magazine, vol. 25, no. 3, pp. 57-67, May 2006.

[22] A. K. Abbas, K. Heimann, K. Jergus, T. Orlikowsky, and S. Leonhardt, "Neonatal non-contact respiratory monitoring based on real-time infrared thermography," BioMed Eng OnLine, vol. 10, pp. 1-17, Oct. 2011. doi: $10.1186 / 1475-925 X-10-93$

[23] C. B. Pereira, X. Yu, M. Czaplik, R. Rossaint, V. Blazek, and S. Leonhardt, "Remote monitoring of breathing dynamics using infrared thermography," Biomed Opt Express, vol. 6, no. 11, pp. 4378-4394, Oct. 2015.

[24] I. Pavlidis, J. Dowdall, N. Sun, C. Puri, J. Fei, and M. Garbey, "Interacting with human physiology," Computer Vision and Image Understanding, vol. 108, no. 1-2, pp. 150-170, Oct. 2007.

[25] A. Zaffaroni, B. Kent, E. O'Hare, C. Heneghan, P. Boyle, G. O'Connell, M. Pallin, P. de Chazal, and W. T. McNicholas, "Assessment of sleepdisordered breathing using a non-contact bio-motion sensor," J Sleep Res, vol. 22, no. 2, pp. 231-236, Apr. 2013.

[26] S. D. Min, J. K. Kim, H. S. Shin, Y. H. Yun, C. K. Lee, and J.-H. Lee, "Noncontact Respiration Rate Measurement System Using an Ultrasonic Proximity Sensor," IEEE Sensors Journal, vol. 10, no. 11, pp. 17321739, Nov. 2010.

[27] V. Hers, D. Corbugy, I. Joslet, P. Hermant, J. Demarteau, B. Delhougne, G. Vandermoten, and J. P. Hermanne, "New concept using Passive Infrared (PIR) technology for a contactless detection of breathing movement: a pilot study involving a cohort of 169 adult patients," J Clin Monit Comput, vol. 27, no. 5, pp. 521-529, Oct. 2013.

[28] G. Shafiq and K. C. Veluvolu, "Surface Chest Motion Decomposition for Cardiovascular Monitoring," Sci Rep, vol. 4, pp. 1-9, May 2014.

[29] M. Steffen and S. Leonhardt, "Non-Contact Monitoring of Heart and Lung Activity by Magnetic Induction Measurement," Acta Polytechnica, vol. 48, no. 3, pp. 72-78, 2008.

[30] S. Roweis, "EM Algorithms for PCA and SPCA," in Advances in Neural Information Processing Systems 10: Proceedings of the 1997 Conference, Cambridge: MIT Press, 1997, pp. 626-632.

[31] A. Lazaro, D. Girbau, and R. Villarino, "Analysis of Vital Signs Monitoring Using An IR-UWB Radar," Progress in Electromagnetics Research, vol. 100, pp. 265-284, 2010.

[32] V. Nguyen, A. Q. Javaid, and M. A. Weitnauer, "Harmonic Path (HAPA) algorithm for non-contact vital signs monitoring with IR-UWB radar," in 2013 IEEE Biomedical Circuits and Systems Conference (BioCAS), 2013, pp. 146-149.

[33] G. Farnebäck, "Two-Frame Motion Estimation Based on Polynomial Expansion," in Image Analysis, J. Bigun and T. Gustavsson, Eds. Springer Berlin Heidelberg, 2003, pp. 363-370.

[34] S. Suzuki, T. Matsui, T. Asao, and K. Kotani, "An investigation using high-precision CCD laser displacement sensor to measure body surface motion induced by heartbeat," Journal of Biomedical Science and Engineering, vol. 5, no. 11, pp. 672-677, Nov. 2012.

[35] S. J. Gordon, K. A. Grimmer, and P. Trott, "Understanding sleep quality and waking cervico-thoracic symptoms," Internet Journal of Allied Health Sciences and Practice, vol. 5, pp. 1-12, Apr. 2007.

[36] D. J. Terbizan, B. A. Dolezal, and C. Albano, "Validity of Seven Commercially Available Heart Rate Monitors," Measurement in Physical Education and Exercise Science, vol. 6, no. 4, pp. 243-247, Dec. 2002. 
Non-Contact Vision-Based Cardiopulmonary Monitoring in Different Sleeping Positions - Supplementary Material

\begin{tabular}{|c|c|c|c|c|c|c|c|c|c|c|c|c|}
\hline \multicolumn{13}{|c|}{ Appendix A1: Respiratory Rate Estimation Results by Participant } \\
\hline \multirow{3}{*}{ Participant } & \multicolumn{6}{|c|}{ RMS Error (breaths/min) } & \multicolumn{6}{|c|}{ Mean Percent Error (\%) } \\
\hline & \multicolumn{6}{|c|}{ Position } & \multicolumn{6}{|c|}{ Position } \\
\hline & Supine & Left & Right & Prone & Occluded & Average & Supine & Left & Right & Prone & Occluded & Average \\
\hline 1 & 1.12 & 0.59 & 0.32 & A & 0.43 & 0.62 & $2.9 \%$ & $1.8 \%$ & $0.8 \%$ & A & $0.9 \%$ & $1.6 \%$ \\
\hline 2 & 0.49 & 2.13 & 2.19 & A & 1.92 & 1.68 & $1.7 \%$ & $4.5 \%$ & $3.2 \%$ & A & $5.6 \%$ & $3.7 \%$ \\
\hline 3 & 0.30 & 0.59 & 1.01 & A & 0.23 & 0.54 & $2.2 \%$ & $2.4 \%$ & $3.6 \%$ & A & $1.6 \%$ & $2.5 \%$ \\
\hline 4 & 0.64 & A & A & A & A & 0.64 & $2.5 \%$ & A & A & A & A & $2.5 \%$ \\
\hline 5 & 0.81 & 0.25 & 0.42 & A & 0.42 & 0.48 & $2.2 \%$ & $1.1 \%$ & $1.5 \%$ & A & $0.9 \%$ & $1.4 \%$ \\
\hline 6 & 0.27 & 0.14 & A & A & 0.84 & 0.42 & $1.0 \%$ & $0.7 \%$ & A & A & $2.0 \%$ & $1.3 \%$ \\
\hline 7 & 0.13 & 0.14 & 0.24 & 0.30 & $1.53^{B}$ & 0.47 & $0.7 \%$ & $0.8 \%$ & $1.2 \%$ & $1.2 \%$ & $5.6 \%^{\mathrm{B}}$ & $1.9 \%$ \\
\hline 8 & $2.32^{\mathrm{B}}$ & $4.16^{\mathrm{B}}$ & $3.99^{B}$ & $2.33^{B}$ & $1.93^{\mathrm{B}}$ & 2.95 & $11.7 \%^{\mathrm{B}}$ & $15.1 \%^{\mathrm{B}}$ & $11.7 \%^{\mathrm{B}}$ & $4.5 \%^{\mathrm{B}}$ & $4.3 \%^{\mathrm{B}}$ & $9.5 \%$ \\
\hline 9 & 0.53 & 0.49 & 0.25 & 0.60 & 0.91 & 0.55 & $2.4 \%$ & $1.7 \%$ & $1.3 \%$ & $2.6 \%$ & $4.1 \%$ & $2.4 \%$ \\
\hline 10 & 0.34 & $1.99^{\mathrm{B}}$ & $3.26^{\mathrm{B}}$ & 0.17 & 0.19 & 1.19 & $1.1 \%$ & $5.3 \%^{\mathrm{B}}$ & $11.3 \%^{\mathrm{B}}$ & $0.9 \%$ & $0.9 \%$ & $3.9 \%$ \\
\hline 11 & 0.30 & 0.16 & 0.55 & 0.49 & 1.25 & 0.55 & $1.4 \%$ & $1.0 \%$ & $1.8 \%$ & $1.5 \%$ & $2.7 \%$ & $1.7 \%$ \\
\hline 12 & 0.14 & 0.14 & 0.35 & 0.74 & 0.48 & 0.37 & $0.9 \%$ & $0.7 \%$ & $1.4 \%$ & $1.8 \%$ & $1.3 \%$ & $1.2 \%$ \\
\hline 13 & 0.14 & 0.13 & 0.30 & 0.15 & 0.16 & 0.18 & $0.6 \%$ & $0.6 \%$ & $1.1 \%$ & $0.7 \%$ & $0.8 \%$ & $0.8 \%$ \\
\hline 14 & 0.15 & 0.26 & $1.60^{\mathrm{B}}$ & 0.38 & 1.80 & 0.84 & $0.6 \%$ & $0.8 \%$ & $4.6 \%^{\mathrm{B}}$ & $1.7 \%$ & $4.1 \%$ & $2.4 \%$ \\
\hline 15 & $3.65^{B}$ & $5.66^{\mathrm{B}}$ & $5.05^{\mathrm{B}}$ & $4.50^{\mathrm{B}}$ & $5.80^{\mathrm{B}}$ & 4.93 & $7.3 \%^{\mathrm{B}}$ & $13.3 \%^{\mathrm{B}}$ & $17.7 \%^{\mathrm{B}}$ & $12.7 \%^{\mathrm{B}}$ & $15.1 \%^{\mathrm{B}}$ & $13.2 \%$ \\
\hline 16 & 0.45 & 1.27 & 0.58 & 0.31 & 0.63 & 0.65 & $2.1 \%$ & $4.2 \%$ & $1.9 \%$ & $1.5 \%$ & $2.2 \%$ & $2.4 \%$ \\
\hline 17 & $2.78^{B}$ & 0.16 & 0.32 & 0.22 & $2.75^{\mathrm{B}}$ & 1.24 & $7.1 \%^{\mathrm{B}}$ & $0.8 \%$ & $1.3 \%$ & $1.0 \%$ & $5.7 \%^{\mathrm{B}}$ & $3.2 \%$ \\
\hline Average & 0.86 & 1.14 & 1.36 & 0.92 & 1.33 & 1.12 & $2.8 \%$ & $3.4 \%$ & $4.3 \%$ & $2.7 \%$ & $3.6 \%$ & $3.4 \%$ \\
\hline \multirow{3}{*}{ Participant } & \multicolumn{6}{|c|}{ \% Estimates Within 1 breath/min } & \multicolumn{6}{|c|}{ \% Estimates Within 0.5 breaths/min } \\
\hline & \multicolumn{6}{|c|}{ Position } & \multicolumn{6}{|c|}{ Position } \\
\hline & Supine & Left & Right & Prone & Occluded & Average & Supine & Left & Right & Prone & Occluded & Average \\
\hline 1 & $96.6 \%$ & $98.9 \%$ & $99.6 \%$ & A & $98.9 \%$ & $98.5 \%$ & $91.5 \%$ & $98.5 \%$ & $99.6 \%$ & A & $98.9 \%$ & $97.1 \%$ \\
\hline
\end{tabular}




\begin{tabular}{|c|c|c|c|c|c|c|c|c|c|c|c|c|}
\hline 2 & $96.6 \%$ & $91.5 \%$ & $95.1 \%$ & A & $88.3 \%$ & $92.9 \%$ & $89.2 \%$ & $86.3 \%$ & $90.2 \%$ & A & $84.0 \%$ & $87.4 \%$ \\
\hline 3 & $98.5 \%$ & $95.8 \%$ & $96.9 \%$ & A & $99.6 \%$ & $97.7 \%$ & $97.4 \%$ & $92.6 \%$ & $95.1 \%$ & A & $94.6 \%$ & $94.9 \%$ \\
\hline 4 & $97.5 \%$ & A & A & A & A & $97.5 \%$ & $95.2 \%$ & A & A & A & A & $95.2 \%$ \\
\hline 5 & $97.0 \%$ & $98.9 \%$ & $99.3 \%$ & A & $99.0 \%$ & $98.5 \%$ & $87.9 \%$ & $93.3 \%$ & $91.3 \%$ & A & $99.0 \%$ & $92.9 \%$ \\
\hline 6 & $97.4 \%$ & $100.0 \%$ & A & A & $97.8 \%$ & $98.4 \%$ & $95.2 \%$ & $99.6 \%$ & A & A & $94.3 \%$ & $96.4 \%$ \\
\hline 7 & $100.0 \%$ & $100.0 \%$ & $98.6 \%$ & $97.9 \%$ & $89.1 \%^{\mathrm{B}}$ & $97.1 \%$ & $100.0 \%$ & $99.6 \%$ & $95.2 \%$ & $95.5 \%$ & $86.8 \%$ & $95.4 \%$ \\
\hline 8 & $69.1 \%^{\mathrm{B}}$ & $62.3 \%^{\mathrm{B}}$ & $77.0 \%^{\mathrm{B}}$ & $91.4 \%^{\mathrm{B}}$ & $92.4 \%^{\mathrm{B}}$ & $78.4 \%$ & $59.6 \%^{\mathrm{B}}$ & $57.0 \%^{\mathrm{B}}$ & $69.6 \%^{\mathrm{B}}$ & $87.3 \%^{\mathrm{B}}$ & $90.2 \%^{\mathrm{B}}$ & $72.7 \%$ \\
\hline 9 & $98.4 \%$ & $98.7 \%$ & $100.0 \%$ & $97.1 \%$ & $95.4 \%$ & $97.9 \%$ & $94.3 \%$ & $96.7 \%$ & $93.6 \%$ & $91.6 \%$ & $84.3 \%$ & $92.1 \%$ \\
\hline 10 & $98.7 \%$ & $90.2 \%^{\mathrm{B}}$ & $76.2 \%^{\mathrm{B}}$ & $100.0 \%$ & $100.0 \%$ & $93.0 \%$ & $98.7 \%$ & $90.2 \%^{\mathrm{B}}$ & $71.0 \%^{\mathrm{B}}$ & $99.7 \%$ & $99.4 \%$ & $91.8 \%$ \\
\hline 11 & $98.9 \%$ & $99.7 \%$ & $96.6 \%$ & $99.0 \%$ & $96.6 \%$ & $98.2 \%$ & $98.6 \%$ & $99.3 \%$ & $90.6 \%$ & $97.8 \%$ & $93.3 \%$ & $95.9 \%$ \\
\hline 12 & $100.0 \%$ & $100.0 \%$ & $98.4 \%$ & $97.2 \%$ & $98.8 \%$ & $98.9 \%$ & $100.0 \%$ & $100.0 \%$ & $92.4 \%$ & $93.8 \%$ & $97.3 \%$ & $96.7 \%$ \\
\hline 13 & $100.0 \%$ & $100.0 \%$ & $96.5 \%$ & $100.0 \%$ & $100.0 \%$ & $99.3 \%$ & $100.0 \%$ & $100.0 \%$ & $93.3 \%$ & $100.0 \%$ & $99.4 \%$ & $98.5 \%$ \\
\hline 14 & $100.0 \%$ & $99.7 \%$ & $90.7 \%^{\mathrm{B}}$ & $98.7 \%$ & $91.4 \%$ & $96.1 \%$ & $100.0 \%$ & $98.7 \%$ & $80.3 \%^{\mathrm{B}}$ & $97.4 \%$ & $88.9 \%$ & $93.0 \%$ \\
\hline 15 & $88.4 \%^{\mathrm{B}}$ & $76.4 \%{ }^{\mathrm{B}}$ & $62.7 \%^{\mathrm{B}}$ & $74.9 \%^{\mathrm{B}}$ & $76.9 \%^{\mathrm{B}}$ & $75.9 \%$ & $85.7 \%^{\mathrm{B}}$ & $75.5 \%^{\mathrm{B}}$ & $58.4 \%^{\mathrm{B}}$ & $68.8 \%{ }^{\mathrm{B}}$ & $70.0 \%^{\mathrm{B}}$ & $71.7 \%$ \\
\hline 16 & $97.6 \%$ & $94.3 \%$ & $97.5 \%$ & $99.7 \%$ & $97.4 \%$ & $97.3 \%$ & $97.3 \%$ & $94.0 \%$ & $93.3 \%$ & $98.8 \%$ & $91.2 \%$ & $94.9 \%$ \\
\hline 17 & $87.9 \%^{\mathrm{B}}$ & $100.0 \%$ & $96.4 \%$ & $100.0 \%$ & $90.5 \%^{\mathrm{B}}$ & $95.0 \%$ & $87.9 \%^{\mathrm{B}}$ & $100.0 \%$ & $94.5 \%$ & $97.2 \%$ & $89.3 \%^{\mathrm{B}}$ & $93.8 \%$ \\
\hline Average & $95.4 \%$ & $94.1 \%$ & $92.1 \%$ & $96.0 \%$ & $94.5 \%$ & $94.4 \%$ & $92.8 \%$ & $92.6 \%$ & $87.2 \%$ & $93.4 \%$ & $91.3 \%$ & $91.5 \%$ \\
\hline
\end{tabular}

${ }^{A}$ Trials not recorded due to technical difficulties or protocol change.

${ }^{B}$ Unwanted motions affected results.

\begin{tabular}{|c|c|c|c|c|c|c|c|c|c|c|c|c|}
\hline \multicolumn{13}{|c|}{ Appendix A2: Heart Rate Estimation Results by Participant } \\
\hline \multirow{3}{*}{ Participant } & \multicolumn{6}{|c|}{ RMS Error (bpm) } & \multicolumn{6}{|c|}{ Mean Percent Error (\%) } \\
\hline & \multicolumn{6}{|c|}{ Position } & \multicolumn{6}{|c|}{ Position } \\
\hline & Supine & Left & Right & Prone & Occluded & Average & Supine & Left & Right & Prone & Occluded & Average \\
\hline 1 & 3.57 & 1.96 & 4.22 & A & 2.48 & 3.06 & $2.9 \%$ & $1.5 \%$ & $3.0 \%$ & A & $2.3 \%$ & $2.4 \%$ \\
\hline 2 & 3.20 & 6.90 & $7.55^{\mathrm{B}}$ & A & 5.10 & 5.69 & $2.4 \%$ & $5.9 \%$ & $5.6 \%^{\mathrm{B}}$ & A & $5.2 \%$ & $4.8 \%$ \\
\hline 3 & 0.58 & $6.74^{\mathrm{B}}$ & $19.68^{B}$ & A & 0.67 & 6.92 & $0.8 \%$ & $3.2 \%^{\mathrm{B}}$ & $7.6 \%^{\mathrm{B}}$ & A & $0.9 \%$ & $3.1 \%$ \\
\hline 4 & 0.74 & A & A & A & A & 0.74 & $0.8 \%$ & A & A & A & A & $0.8 \%$ \\
\hline 5 & 1.42 & 2.73 & $24.92^{C}$ & A & 1.65 & 7.68 & $1.5 \%$ & $2.0 \%$ & $21.9 \%^{\mathrm{C}}$ & A & $1.7 \%$ & $6.8 \%$ \\
\hline
\end{tabular}




\begin{tabular}{|c|c|c|c|c|c|c|c|c|c|c|c|c|}
\hline 6 & 2.32 & 1.45 & A & A & 4.79 & 2.85 & $1.8 \%$ & $1.2 \%$ & A & A & $4.4 \%$ & $2.5 \%$ \\
\hline 7 & 0.86 & 0.86 & 4.84 & 6.70 & 2.88 & 3.23 & $1.4 \%$ & $1.4 \%$ & $4.7 \%$ & $6.1 \%$ & $3.6 \%$ & $3.5 \%$ \\
\hline 8 & $24.26^{\mathrm{D}}$ & $19.46^{\mathrm{D}}$ & $22.71^{\mathrm{D}}$ & $22.72^{\mathrm{D}}$ & $14.27^{\mathrm{D}}$ & 20.69 & $25.1 \%^{\mathrm{D}}$ & $17.5 \%^{\mathrm{D}}$ & $24.6 \%^{\mathrm{D}}$ & $23.5 \%^{\mathrm{D}}$ & $11.2 \%^{\mathrm{D}}$ & $20.4 \%$ \\
\hline 9 & 1.32 & 3.17 & 1.14 & 3.46 & 1.25 & 2.07 & $1.6 \%$ & $2.1 \%$ & $1.7 \%$ & $3.1 \%$ & $1.7 \%$ & $2.0 \%$ \\
\hline 10 & 1.41 & $5.36^{\mathrm{E}}$ & $6.23^{\mathrm{E}}$ & 1.84 & 1.58 & 3.28 & $1.8 \%$ & $3.4 \%^{\mathrm{E}}$ & $5.4 \%^{\mathrm{E}}$ & $2.0 \%$ & $2.2 \%$ & $3.0 \%$ \\
\hline 11 & 1.53 & 3.38 & 4.62 & 12.46 & 2.00 & 4.80 & $1.8 \%$ & $2.2 \%$ & $2.9 \%$ & $6.3 \%$ & $2.1 \%$ & $3.1 \%$ \\
\hline 12 & 0.47 & 0.66 & 0.59 & 5.49 & 0.89 & 1.62 & $0.6 \%$ & $0.7 \%$ & $0.7 \%$ & $2.8 \%$ & $1.0 \%$ & $1.2 \%$ \\
\hline 13 & 4.16 & 1.87 & 1.07 & 4.51 & 1.35 & 2.59 & $1.3 \%$ & $0.9 \%$ & $0.9 \%$ & $3.2 \%$ & $1.3 \%$ & $1.5 \%$ \\
\hline 14 & 0.95 & 1.81 & 6.21 & 11.43 & 4.40 & 4.96 & $1.2 \%$ & $2.3 \%$ & $5.4 \%$ & $13.5 \%$ & $3.3 \%$ & $5.1 \%$ \\
\hline 15 & $7.13^{\mathrm{E}}$ & $10.04^{\mathrm{E}}$ & $13.97^{\mathrm{E}}$ & $17.38^{\mathrm{E}}$ & $12.04^{\mathrm{E}}$ & 12.11 & $5.2 \%^{\mathrm{E}}$ & $7.4 \%^{\mathrm{E}}$ & $14.9 \%^{\mathrm{E}}$ & $16.4 \%^{\mathrm{E}}$ & $10.6 \%^{\mathrm{E}}$ & $10.9 \%$ \\
\hline 16 & 2.36 & 0.67 & 0.98 & 5.38 & 0.84 & 2.05 & $1.0 \%$ & $0.6 \%$ & $0.9 \%$ & $1.7 \%$ & $0.7 \%$ & $1.0 \%$ \\
\hline Average & 3.52 & 4.47 & 8.48 & 9.14 & 3.75 & 5.87 & $3.2 \%$ & $3.5 \%$ & $7.2 \%$ & $7.9 \%$ & $3.5 \%$ & $5.0 \%$ \\
\hline \multirow{3}{*}{ Participant } & \multicolumn{6}{|c|}{ \% Estimates Within $5 \mathrm{bpm}$} & \multicolumn{6}{|c|}{ \% Estimates Within 2.5 bpm } \\
\hline & \multicolumn{6}{|c|}{ Position } & \multicolumn{6}{|c|}{ Position } \\
\hline & Supine & Left & Right & Prone & Occluded & Average & Supine & Left & Right & Prone & Occluded & Average \\
\hline 1 & $93.5 \%$ & $99.3 \%$ & $95.9 \%$ & A & $98.2 \%$ & $96.7 \%$ & $87.8 \%$ & $98.9 \%$ & $86.5 \%$ & A & $81.3 \%$ & $88.6 \%$ \\
\hline 2 & $95.9 \%$ & $78.2 \%$ & $81.4 \%^{\mathrm{B}}$ & A & $78.6 \%$ & $83.5 \%$ & $86.6 \%$ & $70.1 \%$ & $67.4 \%^{\mathrm{B}}$ & A & $59.1 \%$ & $70.8 \%$ \\
\hline 3 & $100.0 \%$ & $93.0 \%{ }^{\mathrm{B}}$ & $95.1 \%^{\mathrm{B}}$ & A & $100.0 \%$ & $97.0 \%$ & $100.0 \%$ & $93.0 \%^{\mathrm{B}}$ & $93.4 \%^{\mathrm{B}}$ & A & $100.0 \%$ & $96.6 \%$ \\
\hline 4 & $100.0 \%$ & A & A & A & A & $100.0 \%$ & $97.1 \%$ & A & A & A & A & $97.1 \%$ \\
\hline 5 & $100.0 \%$ & $98.1 \%$ & $38.8 \%^{\mathrm{c}}$ & A & $99.0 \%$ & $84.0 \%$ & $86.8 \%$ & $88.1 \%$ & $33.0 \%^{\mathrm{c}}$ & A & $84.7 \%$ & $73.1 \%$ \\
\hline 6 & $98.4 \%$ & $96.9 \%$ & A & A & $82.4 \%$ & $92.6 \%$ & $92.3 \%$ & $95.0 \%$ & A & A & $70.1 \%$ & $85.8 \%$ \\
\hline 7 & $100.0 \%$ & $99.3 \%$ & $89.8 \%$ & $82.4 \%$ & $95.3 \%$ & $93.3 \%$ & $97.4 \%$ & $99.3 \%$ & $68.9 \%$ & $75.8 \%$ & $82.1 \%$ & $84.7 \%$ \\
\hline 8 & $35.5 \%^{\mathrm{D}}$ & $50.3 \%^{\mathrm{D}}$ & $28.0 \%^{\mathrm{D}}$ & $28.0 \%^{\mathrm{D}}$ & $70.3 \%^{\mathrm{D}}$ & $42.4 \%$ & $15.1 \%^{\mathrm{D}}$ & $45.0 \%^{\mathrm{D}}$ & $23.9 \%^{\mathrm{D}}$ & $25.4 \%^{\mathrm{D}}$ & $52.5 \%^{\mathrm{D}}$ & $32.4 \%$ \\
\hline 9 & $99.0 \%$ & $98.3 \%$ & $100.0 \%$ & $93.2 \%$ & $100.0 \%$ & $98.1 \%$ & $94.9 \%$ & $91.4 \%$ & $97.8 \%$ & $81.8 \%$ & $93.1 \%$ & $91.8 \%$ \\
\hline 10 & $99.3 \%$ & $90.2 \%^{\mathrm{E}}$ & $79.5 \%^{\mathrm{E}}$ & $97.4 \%$ & $100.0 \%$ & $93.3 \%$ & $95.6 \%$ & $89.2 \%^{\mathrm{E}}$ & $74.3 \%^{\mathrm{E}}$ & $93.8 \%$ & $88.9 \%$ & $88.4 \%$ \\
\hline 11 & $100.0 \%$ & $96.7 \%$ & $96.3 \%$ & $88.5 \%$ & $97.5 \%$ & $95.8 \%$ & $87.5 \%$ & $91.3 \%$ & $84.9 \%$ & $73.9 \%$ & $81.9 \%$ & $83.9 \%$ \\
\hline 12 & $100.0 \%$ & $100.0 \%$ & $100.0 \%$ & $94.8 \%$ & $100.0 \%$ & $99.0 \%$ & $100.0 \%$ & $99.7 \%$ & $100.0 \%$ & $91.7 \%$ & $100.0 \%$ & $98.3 \%$ \\
\hline 13 & $99.3 \%$ & $99.7 \%$ & $99.0 \%$ & $86.1 \%$ & $98.1 \%$ & $96.5 \%$ & $96.6 \%$ & $99.7 \%$ & $98.7 \%$ & $83.7 \%$ & $96.3 \%$ & $95.0 \%$ \\
\hline 14 & $100.0 \%$ & $97.7 \%$ & $83.0 \%$ & $40.2 \%$ & $92.6 \%$ & $82.7 \%$ & $96.4 \%$ & $86.0 \%$ & $70.0 \%$ & $29.4 \%$ & $82.9 \%$ & $72.9 \%$ \\
\hline 15 & $86.7 \%^{\mathrm{E}}$ & $79.7 \%^{\mathrm{E}}$ & $46.6 \%^{\mathrm{E}}$ & $49.2 \%^{\mathrm{E}}$ & $69.0 \%^{\mathrm{E}}$ & $66.2 \%$ & $77.6 \%^{\mathrm{E}}$ & $74.2 \%^{\mathrm{E}}$ & $40.7 \%^{\mathrm{E}}$ & $41.8 \%^{\mathrm{E}}$ & $53.8 \%^{\mathrm{E}}$ & $57.6 \%$ \\
\hline
\end{tabular}




\begin{tabular}{|c|c|c|c|c|c|c|c|c|c|c|c|c|}
\hline 16 & $99.7 \%$ & $100.0 \%$ & $100.0 \%$ & $97.9 \%$ & $100.0 \%$ & $99.5 \%$ & $98.6 \%$ & $98.3 \%$ & $96.9 \%$ & $92.4 \%$ & $97.4 \%$ & $96.7 \%$ \\
\hline Average & $94.2 \%$ & $91.8 \%$ & $81.0 \%$ & $75.8 \%$ & $92.1 \%$ & $87.0 \%$ & $88.1 \%$ & $87.9 \%$ & $74.0 \%$ & $69.0 \%$ & $81.6 \%$ & $80.1 \%$ \\
\hline
\end{tabular}

Note that participant 17 has been excluded from heart rate results due to an irregular heartbeat.

A Trials not recorded due to technical difficulties or protocol change.

${ }^{B}$ Strobing present in recording.

${ }^{C}$ Arm and shoulder blocking upper torso.

${ }^{D}$ Unwanted motions affected results. Speculated that participant anatomy may have also influenced results, but further analysis is required.

${ }^{\mathrm{E}}$ Unwanted motions affected results. 


\section{Appendix A3: Sensitivity Analysis of Algorithm Parameters}

A one-at-a-time sensitivity analysis was performed to determine the sensitivity of algorithm performance to parameter changes.

Results are presented in the table below. Based on this evaluation, further optimization of parameters can yield better accuracy, and better than real time performance ( $>30 \mathrm{fps}$ ) can be realized with comparable accuracy to default parameters. Selection of parameters will depend on application and data quality, but this analysis shows that the algorithm achieves comparable performance for a wide range of parameter values.

\begin{tabular}{|c|c|c|c|c|c|c|c|c|c|c|}
\hline \multirow[b]{2}{*}{ Parameter } & \multirow[b]{2}{*}{ Value } & \multirow[b]{2}{*}{$\begin{array}{c}\text { Speed } \\
\text { (fps) }\end{array}$} & \multicolumn{4}{|c|}{ Respiratory Rate } & \multicolumn{4}{|c|}{ Heart Rate } \\
\hline & & & $\begin{array}{l}\text { RMS Error } \\
\text { (breaths/min) }\end{array}$ & $\begin{array}{l}\quad \% \pm<1 \\
\text { breath/min }\end{array}$ & $\begin{array}{l}\% \pm<0.5 \\
\text { breath } / \mathrm{min}\end{array}$ & $\begin{array}{l}\text { Mean \% } \\
\text { Error }\end{array}$ & $\begin{array}{l}\text { RMS Error } \\
\text { (bpm) }\end{array}$ & $\begin{array}{c}\% \pm<5 \\
\mathrm{bpm}\end{array}$ & $\begin{array}{c}\% \pm<2.5 \\
\text { bpm }\end{array}$ & $\begin{array}{l}\text { Mean \% } \\
\text { Error }\end{array}$ \\
\hline \multirow{4}{*}{$\begin{array}{l}\text { grid size } \\
\text { (in pixels) }\end{array}$} & $20 \times 20, A=48, B=64, P=1$ & 26.44 & 1.12 & $94.5 \%$ & $90.8 \%$ & $3.5 \%$ & 5.83 & $86.7 \%$ & $79.9 \%$ & $5.1 \%$ \\
\hline & $\begin{array}{c}40 \times 40, A=12, B=16, P=8 \\
\text { (default) }\end{array}$ & 26.63 & 1.12 & $94.4 \%$ & $91.5 \%$ & $3.4 \%$ & 5.87 & $87.0 \%$ & $80.1 \%$ & $5.0 \%$ \\
\hline & $80 \times 80, A=6, B=8, P=32$ & 25.74 & 1.17 & $94.4 \%$ & $90.7 \%$ & $3.6 \%$ & 5.71 & $87.3 \%$ & $80.5 \%$ & $4.9 \%$ \\
\hline & $160 \times 160, A=3, B=4, P=128$ & 26.53 & 1.18 & $94.1 \%$ & $90.5 \%$ & $3.7 \%$ & 5.41 & $87.4 \%$ & $80.8 \%$ & $4.8 \%$ \\
\hline \multirow{4}{*}{$\begin{array}{l}\text { points per } \\
\text { cell }(P)\end{array}$} & 4 & 41.83 & 1.12 & $94.5 \%$ & $91.0 \%$ & $3.5 \%$ & 6.27 & $86.5 \%$ & $79.5 \%$ & $5.2 \%$ \\
\hline & 6 & 32.57 & 1.12 & $94.5 \%$ & $90.9 \%$ & $3.5 \%$ & 5.76 & $86.8 \%$ & $80.0 \%$ & $5.0 \%$ \\
\hline & 8 (default) & 26.63 & 1.12 & $94.4 \%$ & $91.5 \%$ & $3.4 \%$ & 5.87 & $87.0 \%$ & $80.1 \%$ & $5.0 \%$ \\
\hline & 10 & 21.78 & 1.12 & $94.4 \%$ & $90.9 \%$ & $3.5 \%$ & 5.78 & $86.9 \%$ & $80.1 \%$ & $5.0 \%$ \\
\hline \multirow{3}{*}{$\begin{array}{c}\text { Discarded } \\
\text { percentiles } \\
(M, N)\end{array}$} & 20 & 24.63 & 1.16 & $94.1 \%$ & $90.5 \%$ & $3.7 \%$ & 6.20 & $86.4 \%$ & $79.4 \%$ & $5.3 \%$ \\
\hline & 25 (default) & 26.63 & 1.12 & $94.4 \%$ & $91.5 \%$ & $3.4 \%$ & 5.87 & $87.0 \%$ & $80.1 \%$ & $5.0 \%$ \\
\hline & 30 & 28.67 & 1.12 & $94.7 \%$ & $91.0 \%$ & $3.5 \%$ & 5.94 & $86.7 \%$ & $80.0 \%$ & $5.1 \%$ \\
\hline \multirow{3}{*}{$\begin{array}{l}\text { Interpolation } \\
\text { factor }\left(L_{H}, L_{R}\right)\end{array}$} & 1 & 26.84 & 1.14 & $94.4 \%$ & $90.8 \%$ & $3.6 \%$ & 5.81 & $86.8 \%$ & $80.0 \%$ & $5.0 \%$ \\
\hline & 2 & 26.69 & 1.14 & $94.4 \%$ & $90.8 \%$ & $3.6 \%$ & 5.93 & $87.1 \%$ & $80.2 \%$ & $5.1 \%$ \\
\hline & 4 (default) & 26.63 & 1.12 & $94.4 \%$ & $91.5 \%$ & $3.4 \%$ & 5.87 & $87.0 \%$ & $80.1 \%$ & $5.0 \%$ \\
\hline \multirow{3}{*}{$\begin{array}{l}\text { Principal } \\
\text { components } \\
\text { for RR }\left(K_{R}\right)\end{array}$} & 3 & & 1.13 & $94.4 \%$ & $90.7 \%$ & $3.6 \%$ & & & & \\
\hline & 5 (default) & & 1.12 & $94.4 \%$ & $91.5 \%$ & $3.4 \%$ & & & & \\
\hline & 7 & & 1.14 & $94.5 \%$ & $90.9 \%$ & $3.5 \%$ & & & & \\
\hline
\end{tabular}




\begin{tabular}{|c|c|c|c|c|c|}
\hline \multirow{3}{*}{$\begin{array}{l}\text { Independent } \\
\text { components } \\
\text { for HR }\left(K_{H}\right)\end{array}$} & 8 & 6.20 & $86.4 \%$ & $79.4 \%$ & $5.3 \%$ \\
\hline & 10 (default) & 5.87 & $87.0 \%$ & $80.1 \%$ & $5.0 \%$ \\
\hline & 12 & 5.90 & $87.4 \%$ & $80.4 \%$ & $5.0 \%$ \\
\hline \multirow{3}{*}{$\begin{array}{l}\text { Min peak } \\
\text { height }(H)\end{array}$} & no threshold & 5.95 & $86.9 \%$ & $80.1 \%$ & $5.1 \%$ \\
\hline & 50th percentile & 5.72 & $87.2 \%$ & $80.4 \%$ & $5.0 \%$ \\
\hline & 90th percentile (default) & 5.87 & $87.0 \%$ & $80.1 \%$ & $5.0 \%$ \\
\hline \multirow{3}{*}{$\begin{array}{l}\text { Max number } \\
\text { of peaks }(\beta)\end{array}$} & 10 & 5.64 & $87.1 \%$ & $80.1 \%$ & $4.9 \%$ \\
\hline & 12 (default) & 5.87 & $87.0 \%$ & $80.1 \%$ & $5.0 \%$ \\
\hline & 15 & 5.98 & $86.9 \%$ & $80.7 \%$ & $5.1 \%$ \\
\hline \multirow{3}{*}{$\begin{array}{l}\text { Frequency } \\
\text { tolerance } \\
\qquad(\omega)\end{array}$} & $4 \mathrm{bpm}$ & 5.90 & $87.0 \%$ & $80.2 \%$ & $5.0 \%$ \\
\hline & $5 \mathrm{bpm}$ (default) & 5.87 & $87.0 \%$ & $80.1 \%$ & $5.0 \%$ \\
\hline & $6 \mathrm{bpm}$ & 5.81 & $86.9 \%$ & $80.2 \%$ & $5.0 \%$ \\
\hline
\end{tabular}

